CONF-9510202--3

UCRL-JC- 119498

PREPRINT

\title{
Model-Based Localization for a Shallow Ocean Experiment
}

\author{
J. V. Candy \\ Lawrence Livermore National Laborator
}

\author{
E. J. Sullivan \\ Naval Undersea Warfare Center
}

This paper was prepared for submission to the

OCEANS '95 MTS/IEEE

October 9-12, 1995, San Diego, CA

July 19, 1995

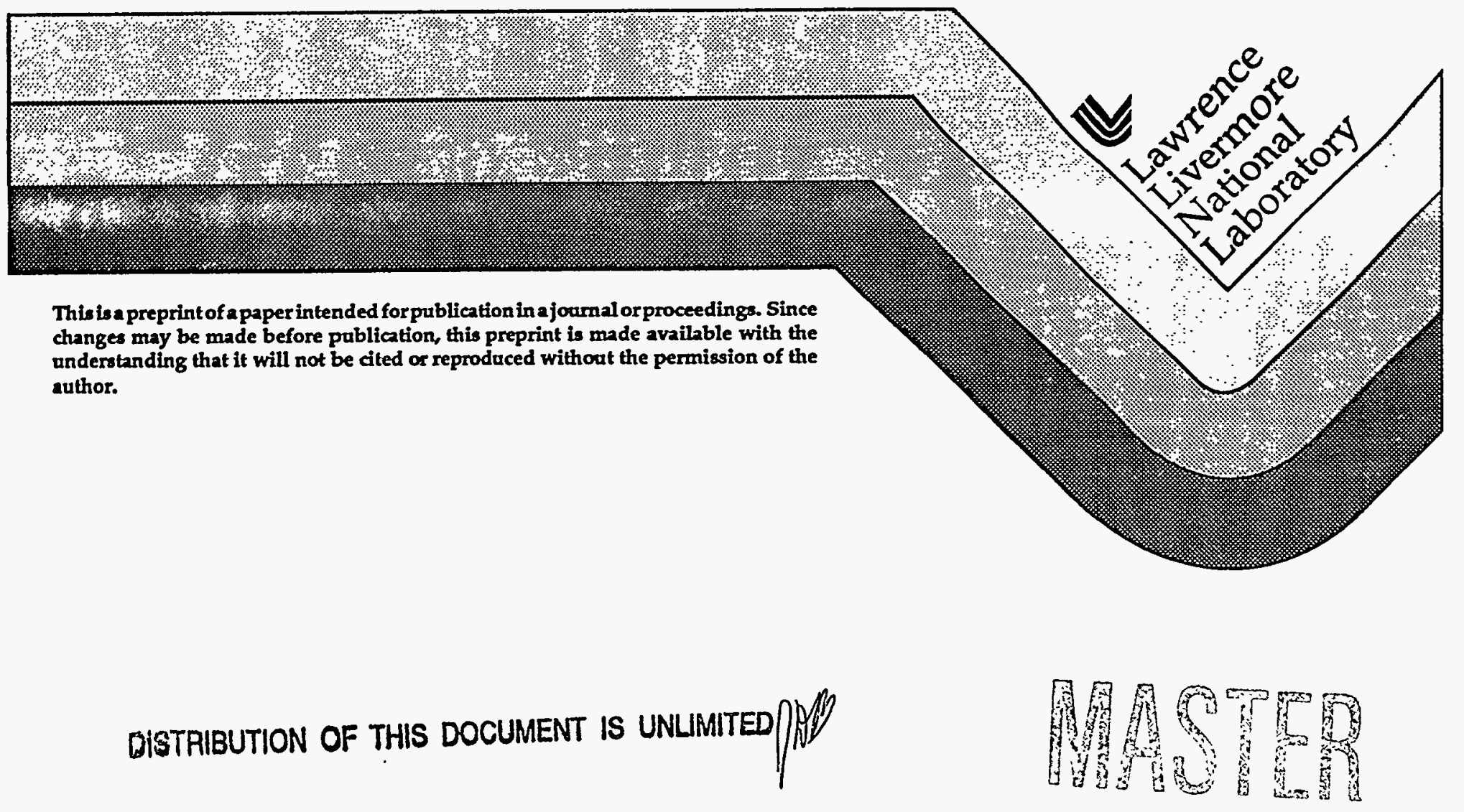


DISCLAIMER

This document was prepared as an account of work sponsored by an agency of the United States Government. Neither the United States Government nor the University of California nor any of their employees, makes any warranty, express or implied, or assumes any legal liability or responsibility for the accuracy, completeness, or usefulness of any information, apparatus, product, or process disclosed, or represents that its use would not infringe privately owned rights. Reference herein to any specific commercial product, process, or service by trade name, trademark, manufacturer, or otherwise, does not necessarily constitute or imply its endorsement, recommendation, or favoring by the United States Government or the University of California. The views and opinions of authors expressed herein do not necessarily state or reflect those of the United States Government or the University of California, and shall not be used for advertising or product endorsement purposes. 


\section{DISCLAIMER}

Portions of this document may be illegible in electronic image products. Images are produced from the best available original document. 


\section{Model-Based Localization for a Shallow Ocean Experiment}

\author{
J.V. Candy \\ Lawrence Livermore National Laboratory \\ Livermore, CA 94550
}

\author{
E.J. Sullivan \\ Naval Undersea Warfare Center \\ Newport, RI 02841
}

\begin{abstract}
A model-based approach is developed to solve the passive localization problem in.ocean acoustics using the state-space formulation. It is shown that the inherent structure of the resulting processor consists of a parameter estimator coupled to a nonlinear optimization scheme. The parameter estimator is designed using an ocean acoustic propagation model in developing the model-besed identifier required for localization.
\end{abstract}

\section{INTRODUCTION}

The detection and localization of an acoustic source has long been the motivation of early sonar systems. With the advent of quieter and quieter submarines due to new manufacturing technologies and the recent prolifieration of diesel powered vessels, the need for more sophisticated processing techniques has been apparent for quite some time. It has often been contemplated that the incorporation of ocean acoustic propagation models into signal processing schemes can offer more useful information necessary to improve overall processor performance and perform the desired detection/localization even under the most hostile of conditions. Model-based techniques offer high expectations of performance, since a processor based on the predicted physical phenomenology that inherently has generated the measured signal must produce a better (minimum error variance) estimate then one that does not. $[1,2]$ However, if the model embedded in this processor is inaccurate or for that matter incorrect, than the model-based processor (MBP) can actually perform worse. Therefore, it is necessary, as part of the MBP design procedure, to estimate/update the model parameters either through separate experiments or jointly (adaptively) while performing the required processing. $[3,4]$

Let us examine the inherent structure of the model-based localizer shown in Figure 1. Here we see that it consists of two distinct parts: a parameter estimator implemented using a modelbased identifier (MBID) and a nonlinear optimizer to estimate the source position. We see that the

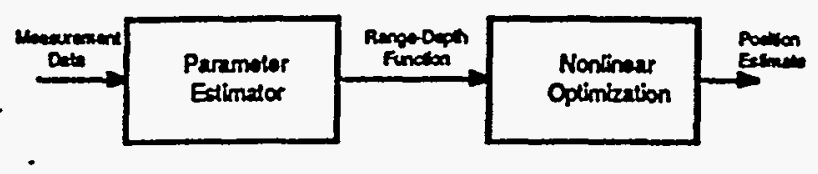

Figure 1: Basic Model-Based Localizer.

main purpose of the parameter estimator is to provide estimates of the inherent localization functions that then must be solved (implicitly) for the desired position. In our application we will show that the parameter estimator (or identifier) will be model-based incorporating the ocean acoustic propagation model. Thus we see that it is, in fact, the MBP or in this case MBID that provides the heart of the model-based localization scheme.

We develop the model-based localizer (MBL) for our ocean acoustic problem and show how it can be realized by utilizing the MBID coupled to a nonlinear optimizer. It will also be shown that the MBID provides an enhanced estimate of the required range-depth function which is then supplied to the optimal position estimator. Next we develop the complete processor and show the structure of the embedded MBID. The MBL is applied to data from a shallow ocean experiment. The results are impressive and clearly show the impact of using the MBID for signal enhancement prior to localization. We summarize our results in the final section.

\section{MODEL-BASED LOCALIZATION}

In this section we develop a model-based localizer for use with an ocean acoustic propagation model. For propagation in a shallow water environment we choose the normal-mode model which can easily be placed in state-space form.

Assuming a horizontally-stratified ocean of depth $h$ with a known source position, we follow the approach discussed in Clay [5] using the separation of variables technique leading to an eigen- 
value equation in $z$ with

$$
\frac{d^{2}}{d z^{2}} \phi_{m}(z)+\kappa_{z}^{2}(m) \phi_{m}(z)=0, m=1, \cdots, M
$$

whose eigensolutions $\left\{\phi_{m}(z)\right\}$ are the so called modal functions and $\kappa_{x}$ is the wavenumber in the z-direction. These solutions depend on the sound speed profile, $c(z)$, and the boundary conditions at the surface and bottom as well as the corresponding dispersion relation given by

$$
\kappa^{2}=\frac{\omega^{2}}{c^{2}(z)}=\kappa_{r}^{2}(m)+\kappa_{x}^{2}(m), \quad m=1, \ldots, M
$$

where $\kappa_{r}, \kappa_{z}$ are the respective wave numbers in the $r$ and $z$ directions with $c$ the depth-dependent sound speed profile and $\omega$ the harmonic source frequency.

For our purpose we are concerned with the estimation of the pressure field, therefore we remove the time dependence, normalize units, and obtain the acoustic preasure propagation model,

$$
p\left(r_{s}, z\right)=\sum_{m=1}^{M} q H_{0}\left(\kappa_{r}(m) r_{s}\right) \phi_{m}\left(z_{s}\right) \phi_{m}(z)
$$

where $p$ is the acoustic pressure; $q$ is the source amplitude; $\phi_{m}$ is the $m^{t h}$ modal function at $z$ and $z_{s} ; \kappa_{T}(m)$ is the horizontal wavenumber associated with the $m^{\text {th }}$ mode; $r_{s}$ is the source range, and $H_{0}\left(\kappa_{r} r_{s}\right)$ is the zeroth-order Hankel function which is the range solution.

The normal-mode solutions can easily be placed in state-space form and we refer the interested reader to Refr. 3 for the detailed theory. This approach leads to a Gauss-Markov model which includes the second order statistics for the measurement and modal noise fields. By using the Hankel function $H_{0}\left(\kappa_{r} r_{s}\right)$ for range, we reduce the state-space model to that of "depth only" and the Gauss-Markov representation for this model is given by

$$
\frac{d}{d z} \phi(z)=A(z) \phi(z)+B(z) \mathbf{u}(z)+w_{\phi}(z)
$$

where $u(z)$ is the source function, $A(z):=\operatorname{diag}\left[A_{1}(z) \cdots A_{M}(z)\right]$ and

$$
A_{m}(z)=\left[\begin{array}{cc}
0 & 1 \\
-\kappa_{z}^{2}(m) & 0
\end{array}\right] \quad m=1, \cdots, M
$$

Since our vertical array spatially samples the pressure-field at each sensor location $z_{l}$, the corresponding measurement model given by

$$
p\left(r_{\ell}, z_{\ell}\right)=C^{T}\left(r_{\ell}, z\right) \phi\left(z_{\ell}\right)+v\left(z_{\ell}\right)
$$

where

$$
C^{T}\left(r_{s}, z\right)=\left[\beta_{1}\left(r_{s}, z_{s}\right) 0\left|\beta_{2}\left(r_{s}, z_{s}\right) 0\right| \cdots \mid \beta_{M}\left(r_{s}, z_{s}\right) 0\right]
$$

with $\beta_{m}\left(r_{s}, z_{s}\right)=\frac{g \phi_{m}\left(x_{s}\right)}{\int_{0}^{h} \phi_{m}^{2}(x) d x} H_{0}\left(k_{r}(m) r_{s}\right)$. The random noise vectors $w_{\phi}$ and $v$ are assumed gaussian, zero-mean with respective covariance matrices, $R_{w_{\phi} w_{\phi}}$ and $R_{v v \text {. }}$

The localization problem solution evolves from the measurement equation of the Gauss-Markov model where we can write the sampled pressurefield in terms of range-depth dependent terms as

$$
p\left(r_{\varepsilon}, z_{l}\right)=\sum_{m=1}^{M} \beta_{m}\left(r_{l}, z_{l}\right) \phi_{m 1}\left(z_{l}\right)+\mathrm{v}\left(z_{\ell}\right)
$$

For the two-dimensional localization problem, we can decompose the pressure measurement further as

$$
p\left(r_{s}, z_{\ell}\right)=\sum_{m=1}^{M} \gamma_{m} \theta_{m}\left(r_{s}, z_{s}\right) \phi_{m 1}\left(z_{\ell}\right)+v\left(z_{\ell}\right)
$$

where $\gamma_{m}$ represents the knoun function and $\theta_{m}\left(r_{s}, z_{s}\right)$ the unknown function of position. Equating these functions with $\beta_{m}$ above we have that

$$
\gamma_{m}=\frac{q}{\int_{0}^{h} \phi_{m}^{2}(z) d z}
$$

and

$$
\theta_{m}\left(r_{s}, z_{s}\right)=H_{0}\left(k_{r}(m) r_{s}\right) \phi_{m}\left(z_{s}\right)
$$

an implicit, separable function of $r_{*}$ and $z_{*}$ which we will call the source range-depth function. With these definitions in mind it is now possible to define (simply) the model-based localization problem as: GIVEN a set of noisy pressure-field and sound speed measurements, $\left[\left\{p\left(r_{s}, z_{l}\right)\right\},\left\{c\left(z_{l}\right)\right\}\right]$ and the normal-mode propagation model, FIND the "best" (minimum error variance) estimate of the source position $\left(r_{s}, z_{s}\right)$, that is, find $\hat{r}_{s}$ and $\hat{z}_{a}$.

In order to solve this problem we must first estimate the "unknown" rango-depth function $\theta_{m}\left(r_{s}, z_{s}\right)$ from the noisy pressure-field measurements and then use numerical optimization techniques to perform the localization $\left(r_{s}, z_{s}\right)$. We discuss the model-based processor used to perform the required parameter estimation in the next section, here we concentrate on the localization problem and the related range-depth functions.

In the design of a localizer we choose a nonlinear least squares approach.[6] Thus, the optimization problem is to find the source position $\left(r_{s}, z_{s}\right)$ that 
minimizes

$$
J\left(r_{s}, z_{s}\right):=\frac{1}{M} \sum_{m=1}^{M}\left(\theta_{m}\left(r_{s}, z_{s}\right)-H_{0}\left(\kappa_{\tau}(m) r_{s}\right) \phi_{m}\left(z_{s}\right)\right)^{2}
$$

We choose to use a direct search method for our localizer primarily because it requires the mini$\mathrm{mal}$ amount of a-priori information and should slowly converge to the optimum. For an on-line application, more rapidly convergent algorithms requiring a-priori information (gradient and Hessian) should be investigated, [6] but here we use an off-line search to investigate the feasibility of the model-based localization.

The "direct search" localization algorithm follows the polytope method of Nelder-Meade.[6] At each stage of iteration, $N+1$ points, say $\alpha_{1}, \cdots, \alpha_{N+1}$, are retained together with the function of these values, that is,

$\alpha_{n}:=\left(r_{n}, z_{n}\right) \quad J\left(\alpha_{n}\right)$ for $n=1,2, \cdots, N+1$

where the functions are ordered such that

$$
J\left(\alpha_{N+1}\right) \geq J\left(\alpha_{N}\right) \geq \cdots \geq J\left(\alpha_{1}\right)
$$

and constitute the vertices of the polytope. At each iteration, a new polytope is generated producing a new point to replace the "worst" point $\alpha_{N+1}$-the point with the largest function value. If we define $c(\alpha)$ as the centroid of the "best" $N$ vertices $\alpha_{1}, \cdots, \alpha_{N}$ given by

$$
c(\alpha)=\frac{1}{N} \sum_{n=1}^{N} \alpha_{n}
$$

then at the beginning of the $n^{\text {th }}$-iteration a search or trial point is constructed by a single reflection step using

$$
\alpha_{r}=c(\alpha)+\left(c(\alpha)-\alpha_{N+1}\right) \Delta_{r}
$$

where $\Delta_{r}$ is the reflection coeficient $\left(\Delta_{r}>0\right)$. The function is evaluated at $\alpha_{r}$ giving $J\left(\alpha_{r}\right)$ and yielding three possibilities:

- (1) $J\left(\alpha_{1}\right) \leq J\left(\alpha_{r}\right) \leq J\left(\alpha_{N}\right)$ and therefore $\alpha_{r} \longrightarrow \alpha_{N+1}$; or

- (2) $J\left(\alpha_{r}\right)<J\left(\alpha_{1}\right)$ and $\alpha_{r} \rightarrow \alpha_{1}$ a new "best" point, since we are minimizing $J$. The direction $\Delta_{r}$, is assumed correct and we then expand the polytope by defining

$$
\alpha_{e}=c(\alpha)+\left(\alpha_{r}-c(\alpha)\right) \Delta_{e}
$$

where $\Delta_{e}$ is the expansion coeficient $\left(\Delta_{e}\right\rangle$ 1). If $J\left(\alpha_{e}\right) \leq J\left(\alpha_{r}\right), \alpha_{e} \longrightarrow \alpha_{N+1}$ otherwise $\alpha_{r} \rightarrow \alpha_{N+1}$; or
- (3) $J\left(\alpha_{r}\right)>J\left(\alpha_{N}\right)$, the polytope is too large and we must "contract" it using

$\alpha_{c}=\left\{\begin{array}{cc}\alpha_{1}+\left(\alpha_{N+1}-\alpha_{1}\right) \Delta_{c} & J\left(\alpha_{r}\right) \geq J\left(\alpha_{N+1}\right) \\ \alpha_{1}+\left(\alpha_{r}-\alpha_{1}\right) \Delta_{c} & J\left(\alpha_{r}\right)<J\left(\alpha_{N+1}\right)\end{array}\right.$

where $\Delta_{c}$ is the contraction coefficient. If $J\left(\alpha_{c}\right)<\min \left\{J\left(\alpha_{r}\right), J\left(\alpha_{N+1}\right)\right\}$, then $\alpha_{c} \longrightarrow$ $\alpha_{N+1}$.

Using the MATLAB Optimization Toolbox [7], we apply the polytope algorithm to a synthesized set of $\left\{\theta_{m}\left(r_{s}, z_{s}\right)\right\}, m=1, \cdots, M$ generated from the true range-depth surface representing the shallow water experiment discussed in the next section. The algorithm converges quite well to the true position and therefore we conclude that this is a wellposed problem with a global optimum solution as long as the unknown functions $\left\{\hat{\theta}_{m}\left(r_{s}, z_{s}\right)\right\}$ can be estimated from the noisy pressure-field measurements.

Before we discuss the details of the MBID, let us see how we can utilize the model-based approach to implement our localizer. From the cost function $J\left(r_{s}, z_{s}\right)$ of Eq. 12, we see that we must have an estimate of the range-depth-function, $\theta_{m}\left(r_{s}, z_{s}\right)$ and this is provided by our MBID. However, we must also have estimates of the associated Hankel function, $H_{0}\left(\kappa_{r} r_{n}\right)$ and the corresponding modal functions evaluated at the current iterate depth, $z_{n}$ as $\phi_{m 1}\left(z_{n}\right)$. The MBID provides us with estimates of these modal functions $\left\{\hat{\phi}_{m 1}\left(z_{\ell}\right)\right\}, \quad m=1, \cdots, M ; \quad l=1, \cdots, L$ at each sensor location (in depth). Since the optimizer requires a finer mesh (in depth) than the modal function estimates at each sensor to perform its search, we use the state-space propagator to generate the estimates at a finer depth sampling interval

$$
\Delta z_{n}:=\frac{\Delta z_{l}}{p} \quad p \in I
$$

Thus for a given value of "search" depth $z_{n}$, we find the closest available depths from the estimator (array geometry) to bracket the search depth, $z_{\ell-1}<z_{n}<z_{\ell}$, and use the lower bound $z_{\ell-1}$ to select the initial condition vector for our propagator. We then propagate the modal function at the finer $\Delta z_{n}$ to obtain the desired estimate at $\hat{\phi}_{m 1}\left(z_{n}\right)$.

Note that the propagator evolves simply by discretizing the differential equation using first differences

$$
\frac{d}{d z} \phi(z) \approx \frac{\phi\left(z_{n}\right)-\phi\left(z_{n-1}\right)}{\Delta z_{n}}
$$


which leads to the corresponding state-space propagator given by

$\hat{\phi}\left(z_{n}\right)=\left[I-\Delta z_{n} A\left(z_{n}\right)\right] \hat{\phi}\left(z_{n-1}\right)$ for $\hat{\phi}\left(z_{n-1}\right)=\phi\left(z_{l-1}\right)$

In this way the state-space propagator is used to provide functional estimates to the nonlinear optimizer for localization, so we see that the MBID of the next section is designed not only to provide estimates of the range-depth function, but also provide enhanced estimates of the modal functions at each required depth iteration, that is

$$
\left[\left\{\hat{\theta}_{m}\left(r_{s}, z_{s}\right)\right\},\left\{\hat{\phi}_{m 1}\left(z_{l}\right)\right\}\right] \longrightarrow\left[\left\{\hat{\phi}_{m}\left(z_{n}\right)\right\},\left(\hat{r}_{s}, \hat{z}_{s}\right)\right]
$$

From an estimation viewpoint, it is important to realize the ramifications of the output of the processor and its relationship to the position estimates. The respective range-depth and modal estimates $\hat{\theta}$ and $\hat{\phi}$ provided by the MBID are minimum variance estimates (approximately). In the case of gaussian noise, they are, if fact, the maximum likelihood (maximum a-posteriori) estimates and therefore the corresponding maximum likelihood ineariance theorem guarantees that the solutions for the $\left(r_{a}, z_{s}\right)$ are also the maximum likelihood estimates of position.

\section{MBL EXPERIMENT DESIGN}

In this section we first design the parameter estimator or more appropriately model-based identifier (MBID) which provides the basis of our eventual localizer design. Recall that we must develop the identifier to extract the desired range-depth function of the previous section as well as provide the necessary enhancement required for localization. From our previous work, it is clear the the extended Kalman filter (EKF) identifier will satisfy these constraints nicely. $[3,4,8]$

It is clear from the localization discussion in the previous section that we must estimate the vector source range-depth function $\theta\left(r_{e}, z_{s}\right)$ directly from the measured data as well as the required modal functions. The basic approach we take, therefore, is to realize that at a given source depth these implicit range-depth function is fixed, therefore, we can assume that $\theta\left(r_{s}, z_{s}\right)$ is a constant $(\dot{\theta}=0)$ or a random walk with an discrete Gauss-Markov model given by

$$
\theta\left(r_{s}, z_{l}\right)=\theta\left(r_{s}, z_{l-1}\right)+w_{\theta}\left(z_{l-1}\right)
$$

The underlying model for our ocean acoustic problem therefore becomes the normal mode propagetion model (in discrete form) with an augmented parameter space (ignoring the noise sources):

$$
\begin{array}{ll}
\phi_{m 1}\left(z_{l}\right)= & \phi_{m 1}\left(z_{\ell-1}\right)+\Delta z_{\ell} \phi_{m 2}\left(z_{\ell-1}\right) \\
\phi_{m 2}\left(z_{\ell}\right)= & -\Delta z_{\ell} \kappa_{z}^{2}(m) \phi_{m 1}\left(z_{\ell-1}\right)+\phi_{m 2}\left(z_{l-1}\right) \\
\theta_{1}\left(r_{l}, z_{\ell}\right)= & \theta_{1}\left(r_{l}, z_{l-1}\right) \\
\vdots & \vdots \\
\theta_{M}\left(r_{l}, z_{l}\right)= & \theta_{M}\left(r_{s}, z_{l-1}\right)
\end{array}
$$

with corresponding measurement model as before

$$
p\left(r_{s}, z_{\ell}\right)=\sum_{m=1}^{N} \gamma_{m} \theta_{m}\left(r_{s}, z_{\ell}\right) \phi_{m 1}\left(z_{\ell}\right)+v\left(z_{\ell}\right)
$$

We choose this general representation where the set $\left\{\theta_{m}\left(r_{e}, z_{\ell}\right)\right\}, m=1, \cdots, M$ is the unknown implicit functions of range and depth while the parameters, $\left\{\gamma_{m}\right\}$ represent the known a-priori information that is included in the processor. The basic prediction estimates for the $m^{\text {th }}$ mode are

$$
\begin{aligned}
& \hat{\phi}_{m 1}\left(z_{l} \mid z_{l-1}\right)=\hat{\phi}_{m 1}\left(z_{\ell-1} \mid z_{\ell-1}\right)+\Delta z_{\ell} \hat{\phi}_{m 2}\left(z_{l} \mid z_{l-1}\right) \\
& \hat{\phi}_{m 2}\left(z_{\ell} \mid z_{l-1}\right)=-\Delta z_{\ell} \kappa_{x}^{2}(m) \hat{\phi}_{m 1}\left(z_{l-1} \mid z_{\ell-1}\right) \\
& +\hat{\phi}_{m 2}\left(z_{\ell-1} \mid z_{\ell-1}\right), \quad m=1, \cdots, M \\
& \hat{\theta}_{1}\left(r_{a}, z_{\ell} \mid z_{\ell-1}\right)=\hat{\theta}_{1}\left(r_{a}, z_{l-1} \mid z_{l-1}\right) \\
& \begin{array}{cc}
\vdots & \vdots \\
\hat{\theta}_{M}\left(r_{e}, z_{\ell} \mid z_{\ell-1}\right) & =\hat{\theta}_{M}\left(r_{a}, z_{\ell-1} \mid z_{\ell-1}\right)
\end{array}
\end{aligned}
$$

with the corresponding innovations (parameterized by $\theta$ ) are given by

$e\left(z_{\ell}, \theta\right)=p\left(r_{s}, z_{\ell}\right)-\sum_{m=1}^{M} \gamma_{m} \hat{\theta}_{m}\left(r_{s}, z_{\ell} \mid z_{l-1}\right) \hat{\phi}_{m 1}\left(z_{l} \mid z_{l-1}, \theta\right)$

and the joint modal and parameter estimate correction equations

$$
\begin{array}{ll}
\hat{\phi}\left(z_{\ell} \mid z_{\ell}\right) & =\hat{\phi}\left(z_{\ell} \mid z_{\ell-1}\right)+K_{\phi}\left(z_{\ell}\right) e\left(z_{\ell}, \theta\right) \\
\hat{\theta}\left(r_{s}, z_{l} \mid z_{\ell-1}\right) & =\hat{\theta}\left(r_{s}, z_{\ell} \mid z_{\ell-1}\right)+K_{\theta}\left(z_{\ell}\right) e\left(z_{\ell}, \theta\right)
\end{array}
$$

So we see (simply) how the unknown range-depth parameters are augmented into the EKF identifier algorithm with the underlying normal-mode propagation model to yield the MBID construct. We summarize the detailed structure of the modelbased localizer (MBL) with the MBID in Figure 2. We use the experiment at the Hudson Canyon- a well-known shallow water $(73 \mathrm{~m})$ ocean environment [9]. Here a 23-element vertical array is deployed from the bottom with $2.5 m$ separation to measure the pressure-field. We use the following average horizontal wave numbers: $\{0.208,0.199,0.183,0.175,0.142\} m^{-1}$ for the 5 modes supporting the water column from a 


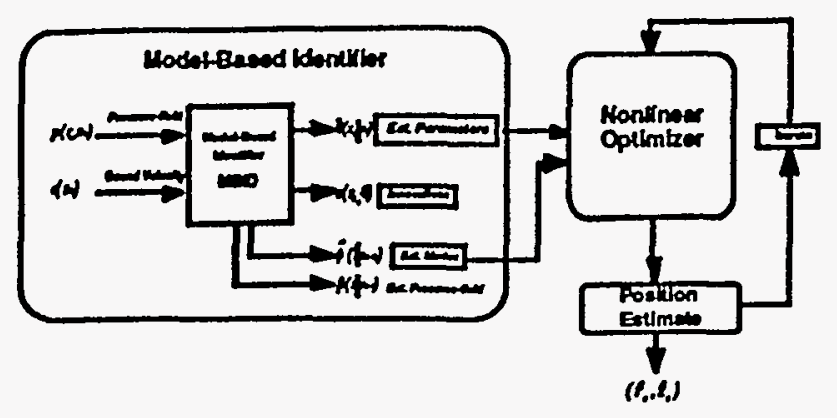

Figure 2: Model-Based Localization (MBL) Processor Structure: (a) MBID. (b) Optimizer.

$36 \mathrm{~m}$ deep, $50 \mathrm{~Hz}$ source at $0.5 \mathrm{Km}$ range. Next we design the MBID using SSPACKPC [10] and investigate the results of the design using the actual experimental hydrophone measurements from the Hudson Canyon. Here we initialize the MBID with the average set of horizontal wave numbers. The resulting estimates are quite reasonable. The results actually appear better than those reported previously for this data set (see Refr. 11) primarily because we have allowed our processor to dynamically adapt (parameter estimator) to the changing parameters. The results for the higher order modes follow those predicted by the model. The reconstructed pressure-field and innovations are also quite reasonable and indicates a "tuned" processor with its zero-mean $\left(1.0 \times 10^{-3}<2.6 \times 10^{-3}\right)$ and white innovations ( $~ 8.3 \%$ out \& WSSR $<\tau$ ). The final parameter estimates with predicted error statistics for this data are also included in Table I for comparison to those previously estimated [11]. We see again that the MBID appears to perform better than the MBP with the augmented identifier simply because the range-depth parameters are "adaptively" estimated, on-line providing \& superior fit to the raw data [8]. Thus, we see that the use of the MBID in conjunction with vertical array measurements enables us to adaptively estimate the required functions as long as we have reasonable estimates to initialize the processor.

Table I. MBID: Range-Depth Parameter Estimation

\begin{tabular}{||c|c|c|}
\hline \multicolumn{3}{|c|}{ Hudson Canyon Experiment } \\
\hline RD Fons & Model Pred & MBID Est/ErT \\
\hline$\theta_{1}$ & 1.000 & $1.015 \pm 0.362$ \\
\hline$\theta_{2}$ & 0.673 & $0.680 \pm 0.364$ \\
\hline$\theta_{3}$ & 0.163 & $0.163 \pm 0.393$ \\
\hline$\theta_{4}$ & 0.166 & $0.166 \pm 0.393$ \\
\hline$\theta_{5}$ & 0.115 & $0.116 \pm 0.364$ \\
\hline
\end{tabular}

Next we consider the application of the MBL to the measured Hudson Canyon data sets. Here we use the MBID to provide estimates of $\left[\left\{\hat{\theta}_{m}\left(r_{a}, z_{s}\right)\right\},\left\{\hat{\phi}_{m 1}\left(z_{l}\right)\right\}\right]$ and we then use the polytope search algorithm along with the statespace propagator of Eq. 22 to provide the localization discussed in the previous section.

We applied the optimizer to the resulting rangedepth parameters estimated by the MBID. The results of the localization are shown in Figure 3. Here we see the range-depth parameter estimates from the MBID, the true values from the simulator and the estimates developed by the optimizer given respectively by the $t, x, 0$, characters on the plots. The corresponding meansquared errors are also shown indicating the convergence of the optimizer after about 40 iterations as well as the actual range-depth search (position iterates) with the true $(500 \mathrm{~m}, 36 \mathrm{~m})$ and estimated $(500.3 m, 36.3 m)$ position estimates shown. The algorithm appears to converge quite readily for a $5 \%$ initial position error. Clearly convergence would be slower for a larger initial position error, but since a global maximum exists and is enhanced after model-based processing the solution should still converge to the correct position. Our-existance of a global maximum also ignores the existance of convergence zones in the ocean medium which could also cause range ambiguities. Further studies will be required to resolve some of these issues including that of a more sophisticated optimizer. Next we designed the MBL for the experimenal measurement data with the localization shown in Figure 4. Here we again see the rangedepth parametric fit, mean-squared error convergence after about 30 iterations and the localization results of $(500.2 \mathrm{~m}, 35.7 \mathrm{~m})$. It appears that the MBL is able to perform quite well over this data set.

To understand why we achieve this type of performance, we have to analyze the synthesized range-depth surface. The effect of the MBID is to enhance this noisy surface easily enabling the optimizer to converge to the correct position. The effect of the MBID leads very closely to the true depth function estimate, since the modal function estimates are quite good. Thus, we can think of the MBID as providing the necessary enhancements in SNR as well as decreasing the dimensionality of the search space to that of the modal space by using the estimated range-depth parameters from the MBID as the "raw" measurement data input (along with the modal estimates) to the polytope optimizer. 


\section{DISCUSSION}

In this paper we have developed an on-line, adaptive, model-based solution to the localization problem, that is, a source position location estimation scheme based on coupling the normal-mode propagation model to a functional model of position. The algorithm employed was the nonlinear extended Kalman filter identifier/parameter estimator coupled to a direct search optimizer using the polytope approach.

It was shown that the model-based localizer follows quite naturally from the model-based identifier. The results of applying the MBL scheme to a simulation of the Hudson Canyon experiment as well as some of the raw experimental data were quite good. In both cases the localizer was able to achieve less than \& $1 \%$ relative pasition error when the initial guesses were within $5 \%$ of the actual position. The feasibilty of the MBL on both simulated and measured data has been demonstated by this work.

\section{References}

[1] A. Jazwinski, Stochastic Processes and Filtering Theory. New York:Academic Press, 1970.

[2] J. V. Candy, Signal Processing: The Model Based Approach. New York:McGraw-Hill, 1986.

[3] J. V. Candy and E. J. Sullivan. "Ocean acoustic signal processing: a model-based approsch." J. Acoust. Soc. Am., 92, (12), 31853201, 1992.

[4] C. S. Clay, and H. Medwin, Acoustical Oceanography. New York:Wiley, 1977.

[5] P.E. Gill, W. Murray and M.H. Wright, Practical Optimization. New York:Academic Press, 1981.

[6] A. Grace, Optimization toolbox for use with MATLAB, Boston: The MathWorks, 1992.

[7] L. Ljung, "Asymptotic behavior of the extended Kalman filter as a parameter estimator for linear systems," IEEE Trans. Auto. Control, AC-24, 36-50, 1979.

[8] J. V. Candy and E.J. Sullivan "Model-based identification: an adaptive approach to ocean acoustic processing.", submitted to IEEE Trans. Ocean Eng., 1994.

[9] W. Carey, J. Doutt and L. Maioco, "Shallow water transmission measurements taken on the New Jersey continental shelf", J. Acoust. Soc. Am., 89, 1981(A), 1991.
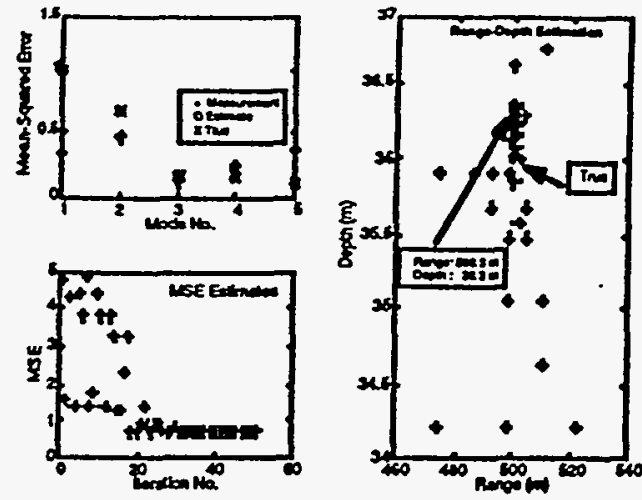

Figure 3: Hudson Canyon Simulation (OdB) Localization: (a ) Estimated Range-Depth Parameters. (b) Mean-Squared Error. (c) Localization: True and Estimated $(500.3 \mathrm{~m}, 36.3 \mathrm{~m})$
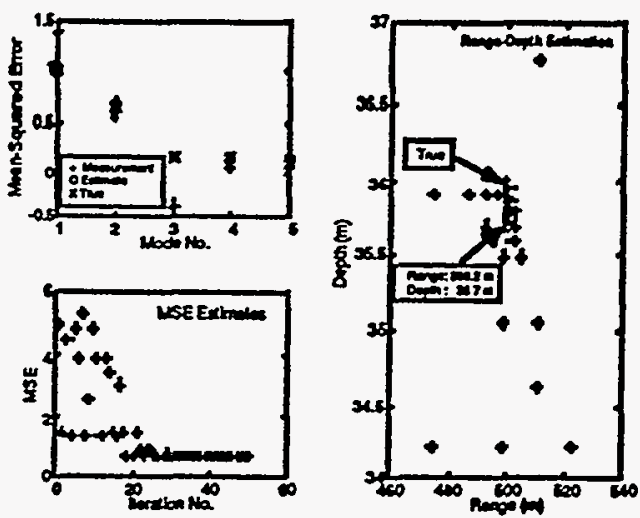

Figure 4: Hudson Canyon Experiment Localization: (a ) Estimated Range-Depth Parameters. (b) Mean-Squared Error. (c) Localization: True and Estimated $(500.2 \mathrm{~m}, 35.7 \mathrm{~m})$

[10] J.V. Candy and P. M. Candy, "SSPACK.PC: A model-based signal processing package on - personal computers," DSP Applic., 2,(3), 3342, 1993.

[11] J.V. Candy and E.J. Sullivan. "Model-based processor design for a shallow water ocean acoustic experiment", J. Acoust. Soc Am., 95, (4) 2038-2051, 1994.

Acknowledgements This work received partial support from the Office of of Naval Research, ONR and the LLNL under the auspices of the U'. S. DOE contract No. W-7405-ENG-48. We acknowledge the motivation and support of Dr. $R$. Doolittle, Dr. T. Goldsberry and Cmdr. M. Shipley of the ONR, and Dr. W. Carey of ARPA for the Hudson Canyon data. 